\title{
Ultrafast processes in photosynthetic light-harvesting
}

\author{
Emilie Wientjes $^{1} \cdot$ Petar Lambrev ${ }^{2}$
}

Published online: 28 April 2020

(c) Springer Nature B.V. 2020

Photosynthesis powers nearly all life on Earth. This tremendously important process starts with the absorption of sunlight by photosynthetic pigments. The excitation energy flows from one pigment to another until it is trapped in the reaction center where charge separation takes place. Photosynthetic pigments are carotenoids, (bacterio)chlorophylls and bilins, which are coordinated by proteins in light-harvesting complexes (Lhcs). The Lhcs are connected with a reaction center to form an active photosynthetic unit. The reaction centers are embedded in a membrane of photosynthetic bacteria, cyanobacteria, (micro)algae and plants (Blankenship 2014). In this Special Issue, we bring together a range of articles which investigate ultrafast processes in photosynthetic light-harvesting from single pigments to intact cyanobacterium cells. A range of experimental techniques, including two-dimensional electronic spectroscopy, fs transient absorption spectroscopy and time-resolved fluorescence, as well as theoretical calculations are employed to increase our understanding of the light-harvesting process.

Using fs transient absorption spectroscopy Staleva-Musto et al. (2019) show how non-conjugated acyl-oxy groups can influence the excited state dynamics of carotenoids by disturbing the electron distribution in the $S_{1}$ /intramolecular charge-transfer state and tuning its charge-transfer character. Next, we move one level up to the excitation energy transfer between two interacting pigments. Although dynamic electronic coherence has been suggested to increase the efficiency of excitation energy transfer between pigments (Engel et al. 2007), recent evidence indicates that the electronic dephasing is too fast for electronic coherences to play a significant role in exciton transfer (Cao et al. 2020). Instead in the work of Duan et al. (2020), the effect of intramolecular vibrations on enhancing energy transfer is investigated by a

Emilie Wientjes

emilie.wientjes@wur.nl

1 Laboratory of Biophysics, Wageningen University, Wageningen, The Netherlands

2 Biological Research Centre, Szeged, Hungary numerically exact hierarchy equation of motion approach. They show that an underdamped vibrational mode which is weakly coupled to the dimer fuels a faster transfer of excitation energy, illustrating that long-lived vibrations can, in principle, enhance energy transfer, without involving longlived electronic coherence. The next level of complexity is light-harvesting complexes coordinating multiple pigments. Two-dimensional electronic spectra provide unique ways to track the energy transfer dynamics in light-harvesting complexes. Kramer and Rodriguez (2019) show by detailed theoretical calculations how two-dimensional electronic spectra of the Fenna-Matthews-Olson complex are affected by rotational and conformational disorder of the chromophores. In the succeeding article, Niedzwiedzki et al. (2019) use transient absorption to investigate how reconstitution of non-native (bacterio)chlorophylls affects excitation energy transfer between the naturally bound carotenoid spheroidene and artificially substituted pigments in the B800 site of lightharvesting 2 (LH2).

While the aim of light-harvesting complexes is to absorb sunlight, the amount of energy harvested at midday can largely exceed the capacity of photosynthesis and should be dissipated safely (Demmig-Adams and Adams 2000). For land plants and algae, it has been suggested that a major part of the excited state energy dissipation in the so-called "nonphotochemical quenching" (NPQ) is located in lightharvesting complex II (LHCII) antenna. Quenching in LHCII aggregates and crystals, in vitro models for NPQ, is investigated by time-resolved fluorescence at various temperatures by Ostroumov et al. (2020). Based on the experimental data combined with quantum chemical calculations, the authors propose a "polarity-switch" model for NPQ, according to which the local environment controls quenching via proton-coupled electron transfer involving chlorophylls 610/611/612. In a related experiment, the chlorophyll charge-transfer state quenching in LHCII was found to be induced by PsbS in proteoliposomes, suggesting that PsbSLHCII interactions trigger the polarity-switch mechanism (Pawlak et al. 2020). 
The final destination of the excitation energy is the reaction center where it should induce charge separation. Timpmann et al. (2020) compare the kinetics of excitation energy trapping by membrane-bound and detergent-purified reaction centers of Rhodobacter sphaeroides under modulating conditions of high hydrostatic pressure. In Photosystem I (PSI) of higher plants, the reaction center is tightly connected to a large antenna system. Russo et al. (2020) investigate why the fluorescence decay of PSI is very similar for open and closed reaction centers. Based on compartmental spectral kinetic modeling of fs transient absorption data, they propose that in closed PSI reaction centers a radical pair state is populated, which decays nonradiatively to the ground state with time constants in the order of 6-8 ps. An increase in the excitation decay rate was observed for PSI trapped in $\mathrm{TiO}_{2}$ mesopores (Szewczyk et al. 2020). This can be caused by increased excitation trapping by the reaction center and/or quenching properties of the $\mathrm{TiO}_{2}$ surface directly interacting with PSI chlorophylls. Akhtar et al. (2020) and Biswas et al. (2020) studied the excitation energy transfer and trapping in isolated PSI, phycobilisomes and intact cells of the cyanobacterium Anabaena PCC 7120 with time-resolved fluorescence spectroscopy. Spectral kinetic modeling of the data indicates how fast the excitation energy flows through the system to be finally trapped by the reaction centers.

The light-harvesting properties of photosynthetic pigments depend on their structure and the relative orientation and distance between pigments. Using X-ray crystallography or electron microscopy, the structures of light-harvesting complexes can be resolved, giving extremely valuable structural data about the pigments and their environment. However, information on the dynamics of the systems at physiologically relevant temperatures is lacking. This Special Issue is concluded with a review of Liguori et al. (2020) on molecular dynamics simulations in photosynthesis. The review explains the principles of molecular dynamics and summarizes how the method can be used to understand the functionality of photosynthetic light-harvesting in a dynamic world.

We hope that this collection of papers addressing Ultrafast Processes in Photosynthetic Light-Harvesting at different levels of complexity contribute to our understanding of the process, which could in the future lead to improved light-harvesting in artificial and natural systems. Finally, we would like to cordially thank all authors and referees who contributed to this Special Issue and Prof. T.M. Bricker, Editor in Chief of Photosynthesis Research, for his advice and support.

\section{References}

Akhtar P, Biswas A, Petrova N, Zakar T, van Stokkum IHM, Lambrev PH (2020) Time-resolved fluorescence study of excitation energy transfer in the cyanobacterium Anabaena PCC 7120. Photosynth Res. https://doi.org/10.1007/s11120-020-00719-w

Biswas A, Huang X, Lambrev PH, van Stokkum IHM (2020) Modelling excitation energy transfer and trapping in the filamentous cyanobacterium Anabaena variabilis PCC 7120. Photosynth Res. https://doi.org/10.1007/s11120-020-00723-0

Blankenship RE (2014) Molecular mechanisms of photosynthesis. Wiley, Hoboken

Cao J, Cogdell RJ, Coker DF, Duan HG, Hauer J, Kleinekathofer U, Jansen TLC, Mancal T, Miller RJD, Ogilvie JP, Prokhorenko VI, Renger T, Tan HS, Tempelaar R, Thorwart M, Thyrhaug E, Westenhoff S, Zigmantas D (2020) Quantum biology revisited. Sci Adv 6(14):eaaz4888. https://doi.org/10.1126/sciadv.aaz4888

Demmig-Adams B, Adams WW (2000) Harvesting sunlight safely. Nature 403(6768):371-373. https://doi.org/10.1038/35000315

Duan HG, Nalbach P, Miller RJD, Thorwart M (2020) Intramolecular vibrations enhance quantum efficiency of excitonic energy transfer. Photosynth Res. https://doi.org/10.1007/s11120-02000742-x

Engel GS, Calhoun TR, Read EL, Ahn TK, Mancal T, Cheng YC, Blankenship RE, Fleming GR (2007) Evidence for wavelike energy transfer through quantum coherence in photosynthetic systems. Nature 446(7137):782-786. https://doi.org/10.1038/ nature 05678

Kramer T, Rodriguez M (2019) Effect of disorder and polarization sequences on two-dimensional spectra of light-harvesting complexes. Photosynth Res. https://doi.org/10.1007/s11120-01900699-6

Liguori N, Croce R, Marrink SJ, Thallmair S (2020) Molecular dynamics simulations in photosynthesis. Photosynth Res. https ://doi.org/10.1007/s11120-020-00741-y

Niedzwiedzki DM, Swainsbury DJK, Hunter CN (2019) Carotenoidto-(bacterio)chlorophyll energy transfer in LH2 antenna complexes from Rba. sphaeroides reconstituted with non-native (bacterio)chlorophylls. Photosynth Res. https://doi.org/10.1007/ s11120-019-00661-6

Ostroumov EE, Götze JP, Reus M, Lambrev PH, Holzwarth AR (2020) Characterization of fluorescent chlorophyll charge transfer states as intermediates in the excited state quenching of light-harvesting complex II. Photosynth Res. https://doi. org/10.1007/s11120-020-00745-8

Pawlak K, Paul S, Liu C, Reus M, Yang C, Holzwarth AR (2020) On the PsbS-induced quenching in the plant major light-harvesting complex LHCII studied in proteoliposomes. Photosynth Res. https://doi.org/10.1007/s11120-020-00740-Z

Russo M, Petropoulos V, Molotokaite E, Cerullo G, Casazza AP, Maiuri M, Santabarbara S (2020) Ultrafast excited-state dynamics in land plants Photosystem I core and whole supercomplex under oxidised electron donor conditions. Photosynth Res. https ://doi.org/10.1007/s11120-020-00717-y

Staleva-Musto H, Kuznetsova V, Bina D, Litvin R, Polivka T (2019) Intramolecular charge-transfer state of carotenoids siphonaxanthin and siphonein: function of non-conjugated acyl-oxy group. Photosynth Res. https://doi.org/10.1007/s11120-019-00694-x

Szewczyk S, Bialek R, Giera W, Burdzinski G, van Grondelle R, Gibasiewicz K (2020) Excitation dynamics in Photosystem I trapped in TiO2 mesopores. Photosynth Res. https://doi. org/10.1007/s11120-020-00730-1 
Timpmann K, Jalviste E, Chenchiliyan M, Kangur L, Jones MR, Freiberg A (2020) High-pressure tuning of primary photochemistry in bacterial photosynthesis: membrane-bound versus detergent-isolated reaction centers. Photosynth Res. https://doi. org/10.1007/s11120-020-00724-Z
Publisher's Note Springer Nature remains neutral with regard to jurisdictional claims in published maps and institutional affiliations. 